\title{
OCT4 is epigenetically regulated by DNA hypomethylation of promoter and exon in primary gliomas
}

\author{
JINLONG SHI $^{1 *}$, WEI SHI ${ }^{*}$, LANCHUN NI $^{1}, \mathrm{XIDE} \mathrm{XU}^{1}, \mathrm{XING} \mathrm{SU}^{1}$, LIANG XIA $^{1}$, \\ FENG $\mathrm{XU}^{2}$, JIAN $\mathrm{CHEN}^{1}$ and JIANHONG $\mathrm{ZHU}^{2}$ \\ ${ }^{1}$ Department of Neurosurgery, Affiliated Hospital of Nantong University, Nantong, Jiangsu 226001; \\ ${ }^{2}$ Department of Neurosurgery, Huashan Hospital, Fudan University, Shanghai 200040, P.R. China
}

Received January 17, 2013; Accepted April 9, 2013

DOI: $10.3892 /$ or.2013.2456

\begin{abstract}
Glioma is the leading cause of tumor-related mortality in the central nervous system. There is increasing evidence that the self-renewal capacity of cancer cells is critical for the initiation, growth and recurrence of tumors. OCT4 is a transcription factor that plays a key role in regulating the self-renewal ability of embryonic stem cells. DNA methylation is involved in the regulation of OCT4 expression during the development and differentiation of embryonic stem cells and neural stem cells. In the present study, we reported that $O C T 4$ was highly expressed in primary gliomas and its expression levels increased in parallel with pathological grades. BSP analysis showed that the methylation levels of OCT4 gene promoter and exon were significantly reduced in comparison with the normal group and were negatively correlated with OCT4 gene expression in primary gliomas. In vitro, OCT4 gene expression was upregulated following treatment by a demethylation reagent in glioma cell lines. Our findings suggest that OCT4 is epigenetically regulated by DNA hypomethylation in primary gliomas, which may provide evidence for the role of DNA methylation in tumor and may present a new direction for developing more powerful strategies to treat glioma in the clinic.
\end{abstract}

\section{Introduction}

Primary malignant gliomas are diagnosed in 6-7 out of 100,000 people each year. Despite advances in treatment strategies that

Correspondence to: Professor Jian Chen, Department of Neurosurgery, Affiliated Hospital of Nantong University, 19 Xisi Road, Nantong, Jiangsu 226001, P.R. China

E-mail: ntshij@163.com

Professor Jianhong Zhu, Department of Neurosurgery, Huashan Hospital, Fudan University, 12 Wulumuqi Zhong Road, Shanghai 200040, P.R. China

E-mail: jl.shi@foxmail.com

*Contributed equally

Key words: glioma, OCT4, hypomethylation combine surgery with radiotherapy and chemotherapy, these tumors remain one of the most fatal diseases (1). The genesis mechanisms of gliomas remain unknown.

It was hypothesized in previous studies that the development and prevalent resistance of brain tumor is attributed to brain tumor stem cells (BTSCs), which are a small population of tumor cells within the heterogeneous tumor mass with stemlike characteristics (2). According to this hypothesis, BTSCs sustain the long-term growth of brain tumor and are responsible for tumor recurrence following conventional treatments. Current treatments combining surgery and chemoradiotherapy are not able to eliminate BTSCs (3-5); therefore, new treatments are required to more efficiently target BTSCs. It is necessary to thoroughly understand the molecular and cellular mechanisms underlying the self-renewal and differentiation of BTSCs.

OCT4, also known as OCT3 or POU5F1, is a member of the POU (Pit, Oct, Unc) transcription factor family encoded by the POU5F1 gene (6p2113) and was first identified in 1990 (6). The expression of OCT4 is restricted to pluripotent stem cells and is downregulated when differentiation is initiated during embryonic development. OCT4 is regarded as a gatekeeper at early mammalian development (7) and regulates the selfrenewal and pluripotency of human embryonic stem cells (ESCs) $(8,9)$. OCT4 plays an important role in maintaining cellular plasticity and promoting the self-renewal and proliferation ability of stem cells (7). In addition, OCT4-positive cells identified in cancer may represent cancer stem cells (CSCs) and account for the maintenance and propagation of tumors $(10,11)$. In primary glioma, OCT4 is highly expressed and increases in parallel with glioma grading, indicating that the reactivation of OCT4 or other stem cell genes may help to maintain the status of BTSCs and enhance the malignancy of high-grade gliomas (12-14). These findings provide evidence of OCT4 for the stem cell theory on tumorigenesis. However, no study has reported the potential mechanism of how OCT4 expression is upregulated with glioma grading in primary gliomas.

Epigenetic regulation, which refers to the alteration in the expression of heritable genes due to the changes in non-DNA sequences, is one of the crucial mechanisms in the control of gene transcription (15). DNA methylation is a mechanism extensively investigated in this field. The methylation of the $\mathrm{CpG}$ island at the promoter usually leads to the downregulation 
of gene expression. Higher level methylation predicts lower transcription activity $(16,17)$. The aberrant DNA methylation pattern can alter gene transcription that is etiologically linked to the formation of cancer (18-20). It was believed that the epigenetic regulation of the chromatin state plays an important role in the control of OCT4 expression (21). DNA methylation of OCT4 gene regulatory regions and histone modifications contribute to the silencing of the OCT4 gene during the differentiation and embryo development of ESCs in mouse and human (22-24). In addition, OCT4 expression to the germ line cells and the early embryo cells may also be mediated by DNA methylation and the mouse ortholog OCT4 is more strongly methylated in differentiated cells than in ESCs (25). OCT4 gene methylation has been studied extensively, but little is known about its DNA methylation at the promoter and exon in primary glioma and its function in tumorigenesis.

Therefore, we hypothesized that DNA methylation may be involved in the regulation of OCT4 expression during the occurrence and development of primary glioma. In this study, we evaluated the expression and the methylation status of OCT4 in 24 patients with primary gliomas and further analyzed their relationship. In vitro, we maintained and treated glioma cell lines with a demethylation reagent to further detect the OCT4 expression.

\section{Materials and methods}

Patient selection and sampling. All the investigations described in this study were conducted after informed consent was obtained and in accordance with an institutional review board protocol approved by the ethics committee at the Affiliated Hospital of Nantong University. A total of 24 patients with primary glioma were recruited from the Department of Neurosurgery, Affiliated Hospital of Nantong University, from January 2009 to December 2011. Pathological findings were determined by more than 2 pathologists and classified according to the WHO classification standard. There were 12 low- (WHO Grade II) and 12 high-grade tumors (WHO Grade III and IV). The glioma tissues were collected and fixed in $10 \%$ formaldehyde followed by embedding in paraffin. In addition, a fraction of samples was placed into liquid nitrogen $\left(-70^{\circ} \mathrm{C}\right)$ for use.

Glioma cells and demethylation treatment. Two human glioma cell lines, U87MG and U251MG, were purchased from the Shanghai Cell Institute of the Chinese Academy of Sciences. Both cell lines were maintained in Dulbecco's Modified Eagle's Medium; Nutrient Mixture F-12 (DMEM/F12) supplemented with $10 \%$ heat-inactivated fetal bovine serum (FBS), $100 \mathrm{U} / \mathrm{ml}$ penicillin and $100 \mu \mathrm{g} / \mathrm{ml}$ streptomycin (all from Gibco). Cells were incubated at $37^{\circ} \mathrm{C}$ in a humidified atmosphere of $5 \%$ $\mathrm{CO}_{2}$. Demethylation with 5-Aza-2'-deoxycytidine; when cell cultures reached $50 \%$ confluence, they were treated with 5-Aza-2'-deoxycytidine (A3656; Sigma-Aldrich, St. Louis, $\mathrm{MO}, \mathrm{USA}$ ) at the final concentration of $10 \mathrm{nM}$, respectively, for 3 days.

RNA isolation and real-time PCR. RNA expression levels of OCT4 were determined using quantitative real-time PCR with GAPDH as positive controls. Total mRNA was isolated from glioma specimens and cell lines using mRNA Isolation Kit (Roche, UK) following the manufacturer's instructions. The concentration and purity of mRNA was determined by ultraviolet spectrophotometry. Isolated mRNA (100 ng) from each sample was transcribed to complementary DNA (cDNA) using a First-Strand cDNA Synthesis Kit (Roche), which was then used as a template for quantitative real-time PCR.

Primers used for OCT4 were: 5'-TATTCAGCCAAACGAC CATCT-3' (sense) and 5'-TCAGCTTCCTCCACCCACTT-3' (antisense); for GAPDH, primers were: 5'-GGAAAGCTGT GGCGTGAT-3' (sense) and 5'-AAGGTGGAAGAATGGGA GTT-3' (antisense). The primers were designed using Primer 5.0 software and manufactured by TIB Molbiol. A $20 \mu \mathrm{l}$ reaction, which included $2 \mu \mathrm{l}$ of cDNA template, $2 \mu \mathrm{l}$ of forward and reverse primer, $6 \mu 1$ DEPC $\mathrm{H}_{2} \mathrm{O}$ and $10 \mu \mathrm{l}$ SYBR-Green Mix (QPK-201, Toyobo), was conducted using the ABI Prism 7500 Sequence Detection System (Applied Biosystems). PCRs of each template were performed in duplicate in a 96-well plate. The thermal cycling conditions included an initial denaturation step at $95^{\circ} \mathrm{C}$ for $5 \mathrm{~min}$ and 40 cycles at $95^{\circ} \mathrm{C}$ for $10 \mathrm{sec}$, at $59^{\circ} \mathrm{C}$ for $15 \mathrm{sec}$ and at $72^{\circ} \mathrm{C}$ for $20 \mathrm{sec}$. The relative fold-change $2^{-\Delta \Delta C T}$ method was used to determine the relative quantitative gene expression compared with GAPDH. The transcription level of target genes observed in calibrating samples was treated as the basal level and given the value 1.0. All PCR reactions were performed in triplicate and a negative control was included that contained primers without cDNA.

Western blot analysis. The samples were then homogenized in lysis buffer (1\% NP-40, $50 \mathrm{mmol} / \mathrm{l}$ Tris, $\mathrm{pH} 7.5,5 \mathrm{mmol} / \mathrm{l}$ EDTA, $1 \%$ SDS, $1 \%$ sodium deoxycholate, $1 \%$ Triton X-100, $1 \mathrm{mmol} / \mathrm{l} \mathrm{PMSF}, 10 \mathrm{mg} / \mathrm{ml}$ aprotinin and $1 \mathrm{mg} / \mathrm{ml}$ leupeptin; Sigma-Aldrich) and clarified by centrifuging for $20 \mathrm{~min}$ in a microcentrifuge at $4^{\circ} \mathrm{C}$. Following determination of its protein concentration with the Bradford Assay (Bio-Rad, USA), the resulting supernatant (50 $\mu \mathrm{g}$ of protein) was subjected to SDS-polyacrylamide gel electrophoresis (PAGE). The separated proteins were transferred to a polyvinylidine difluoride membrane (Millipore Corp., USA) by a transfer apparatus at $350 \mathrm{~mA}$ for $2.5 \mathrm{~h}$. The membranes were first blocked and then incubated with the primary antibody described above for $2 \mathrm{~h}$ at room temperature. After washing three times, filters were incubated with horseradish peroxidase-conjugated human anti-mouse or anti-rabbit antibodies (Pierce) for $1 \mathrm{~h}$ at room temperature. Immunocomplexes were detected with an enhanced chemiluminescence system (NEN Life Science Products, USA). The western blotting experiments were repeated at least three times.

Immunohistochemistry (IHC). Each glioma was immunohistochemically examined for OCT4 nuclear staining. Specimens were cut serially into $4-\mu \mathrm{m}$ sections. Sections were dewaxed in xylene and rehydrated in graded ethanols. Endogenous peroxidase activity was blocked by immersion in $0.3 \%$ methanolic peroxide for $30 \mathrm{~min}$. Immunoreactivity was enhanced by microwaving and incubating the tissue sections for $10 \mathrm{~min}$ in $0.1 \mathrm{~mol} / \mathrm{l}$ citrate buffer. Primary rabbit polyclonal antibodies OCT4 (1:200; Abcam, USA) were applied and incubated overnight at $4^{\circ} \mathrm{C}$. Secondary Goat Anti-Rabbit immunoglobulin (Invitrogen Life Technologies, USA) was applied and incubated 

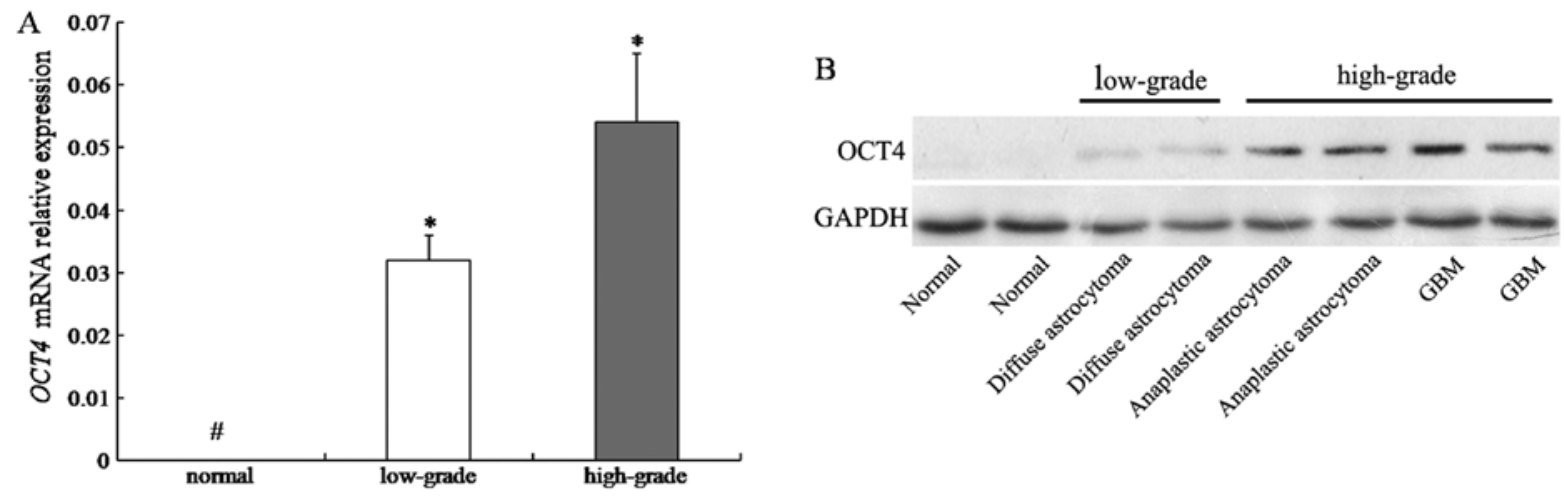

Figure 1. OCT4 is highly expressed in primary gliomas. (A) Relative levels of OCT4 mRNA in high-grade gliomas, low-grade gliomas and normal brain tissues are shown as histograms. OCT4 mRNA expression was quantified by qRT-PCR compared with GAPDH. "P<0.05. (B) Western blot analysis of OCT4 protein levels in high-grade gliomas, low-grade gliomas and normal brain tissues. The blot shows representative results from 2 tissues in different malignant grades and 2 normal brain tissues. GAPDH was used as a loading control.

for $1 \mathrm{~h}$ at room temperature. Some sections were processed with Tris-buffered saline rather than primary antibody and were used as negative controls. Immunocomplexes were visualized by brown pigmentation via a standard 3,3-diaminobenzidine (DAB) protocol. Appropriate positive and negative controls were used. Ten high-power fields were randomly chosen and at least 300 cells were counted per field. Tumors were scored as a percentage of positive cells. The staining procedures were repeated at least three times.

DNA preparation and bisulfite genomic sequencing. Genomic DNA was extracted from frozen tissues by proteinase K digestion and the phenol-chloroform method (26). Sodium bisulfite treatment of the extracted DNA was performed as previously described with some modifications (27). In brief, $10 \mu \mathrm{g}$ DNA in $50 \mu \mathrm{l} \mathrm{TE}$ was incubated with $5.5 \mu \mathrm{l}$ of $0.3 \mathrm{M} \mathrm{NaOH}$ at $37^{\circ} \mathrm{C}$ for $15 \mathrm{~min}$ and at $95^{\circ} \mathrm{C}$ for $2 \mathrm{~min}$ and then subjected to sodium bisulfite chemical treatment (2.4 M sodium metabisulfite; $0.5 \mathrm{mM}$ hydroquinone, $\mathrm{pH}$ 5.0; both from Sigma-Aldrich). Following incubation for $4 \mathrm{~h}$ at $55^{\circ} \mathrm{C}$, the treated DNA was purified using the SK1261 kit (Shenggong Biotechnology Co., China), desulfonated in $0.3 \mathrm{M} \mathrm{NaOH}$ and then neutralized to pH 7.0 using $3 \mathrm{M}$ sodium acetate ( $\mathrm{pH}$ 5.2). The neutralized DNA was then purified using the SK1261 purification kit again, dissolved in TE buffer ( $\mathrm{pH} 8.0)$.

The methylation status of the minimal promoter and part of exon 1 region CpG islands of OCT4 was analyzed $(23,28)$. The primers were designed to amplify the promoter and exon 1 from -234 to +46 for bisulfite genomic sequencing. The forward primer was 5'-GGATTTGTATTGAGGTTTTGGAG-3' and the reverse, 5'-TAACCCATCACCTCCACCAC-3'. Touchdown PCR was then carried out. An initial denaturation at $98^{\circ} \mathrm{C}$ for $4 \mathrm{~min}$ was followed by five PCR cycles of $94^{\circ} \mathrm{C}$ for $45 \mathrm{sec}$, $68^{\circ} \mathrm{C}$ for $45 \mathrm{sec}$ and $72^{\circ} \mathrm{C}$ for $1 \mathrm{~min}$. The PCR was then completed with 35 cycles of $45 \mathrm{sec}$ at $95^{\circ} \mathrm{C}, 45 \mathrm{sec}$ at $58^{\circ} \mathrm{C}$. The amplified products were gel-purified using the SK1261 kit and then subjected to TA-cloning using pUC18-T vector (Shenggong Biotechnology Co.). Ten clones for each case were selected for sequencing using BigDye version 3.1 and analyzed on automated DNA sequence analyzer (ABI Prism 3730; Applied Biosystems, Inc., Foster City, CA, USA). The cytosine or thymine residues at the $\mathrm{CpG}$ sites represented methylated or unmethylated status, respectively.

Statistical analysis. Statistical analysis was performed using SPSS 13.0 for Windows. The Student's independent-sample t-test statistics was used for statistical comparison. The correlation between methylation status and mRNA expression level of OCT4 was tested by Pearson's correlation test. All statistical tests were calculated in two-sided and a P-value $<0.05$ was considered to indicate a statistically significant difference.

\section{Results}

OCT4 is highly expressed in primary gliomas. The primary glioma samples were collected from 24 patients and placed in liquid nitrogen, followed by detection of OCT4 mRNA expression by real-time PCR. Results revealed that expression of OCT4 mRNA was upregulated in all 24 human glioma samples, but no obvious expression was observed in the normal brain samples. Densitometric evaluation of relative expression showed that the level of OCT4 mRNA was significantly higher in high-grade gliomas than in low-grade gliomas $(\mathrm{P}<0.05)$ (Fig. 1A). Western blot analysis confirmed that the level of OCT4 protein was higher in primary high-grade gliomas than in low-grade ones (Fig. 1B).

OCT4 protein is localized in the nuclei of primary glioma cells. The primary glioma tissues were collected from 24 patients and paraffin-embedded sections were obtained for IHC, followed by counting of OCT4-positive cells. OCT4 was expressed in the nuclei of tumor cells and OCT4-positive cells were identified in all glioma samples. The percentage of OCT4-positive cells ranged from 3.79 to $79.67 \%$. The nuclei of most highgrade glioma cells (Fig. 2C and D) were more intensely stained than those of low-grade tumors (Fig. 2A and B), indicating that OCT4 expression in the nuclei of glioma cells was in a gradedependent manner.

Hypomethylation of the OCT4 gene and negative correlation with OCT4 expression in primary glioma. Bisulphate sequencing analysis was employed to examine whether the OCT4 expression 


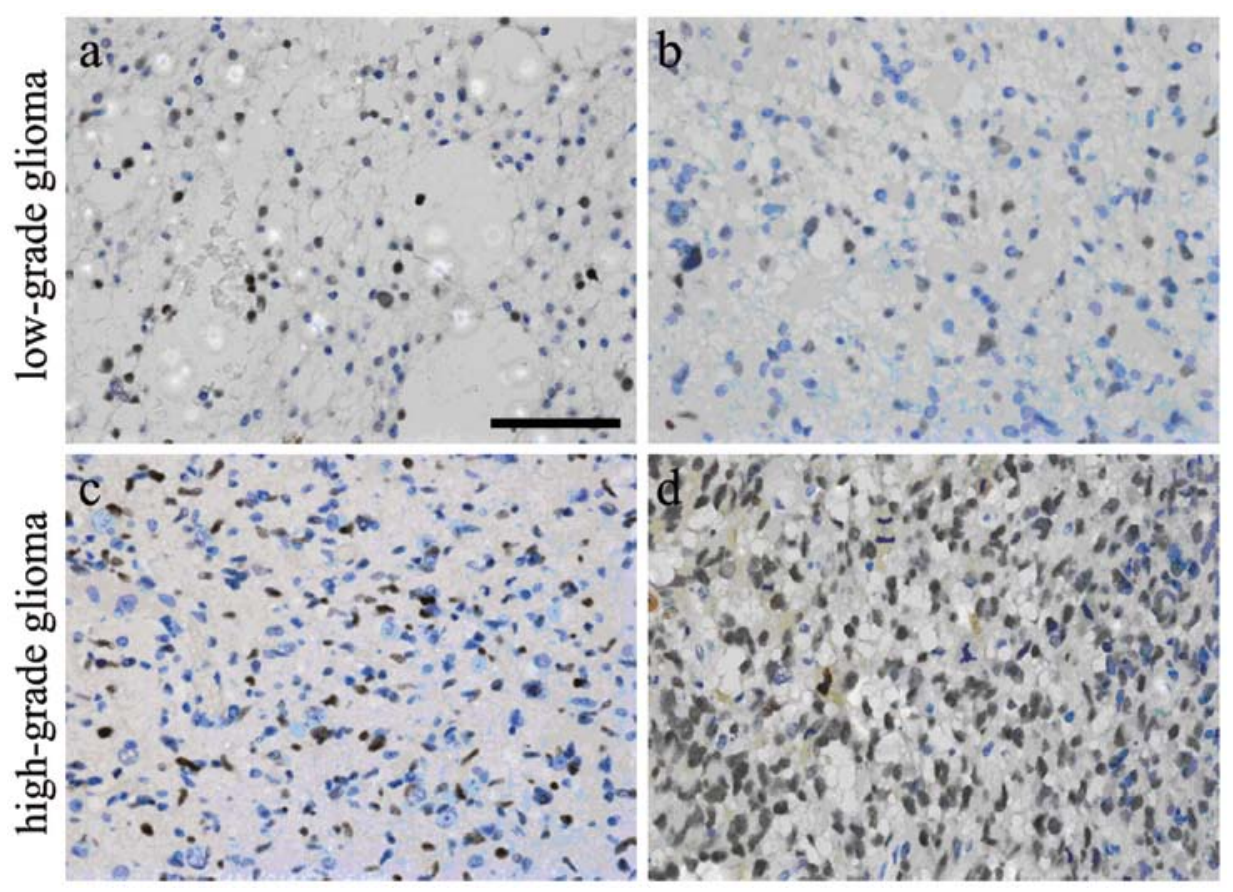

Figure 2. OCT4 expression is localized in the nuclei of primary glioma cells. Immunohistochemical analysis showed that OCT4 expression in paraffinembedded sections from low-grade (A and B) and high-grade (C and D) gliomas. (A-D) OCT4 expression is primarily localized in the nuclei of tumor cells (brown). Scale bar $=100 \mu \mathrm{m}$.
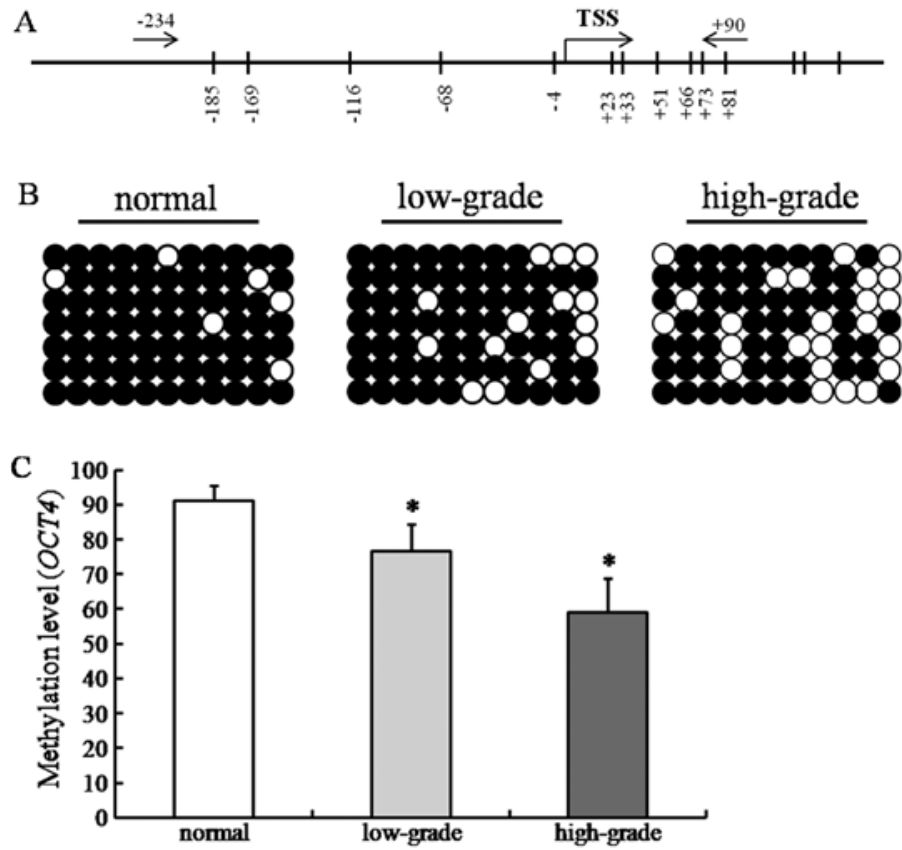

Figure 3. Reduction of DNA methylation of the OCT4 gene. (A) DNA methylation profile of 11 CpG dinucleotides (-234 to +90) located including 5 in the minimal promoter region and 6 in the exon region. (B) Analysis of DNA methylation at 11 known differentially methylated CpG sites in the OCT4 -234 to +90 region with DNA from normal tissues, low-grade gliomas and high-grade gliomas. Black and white circles represent methylated and unmethylated sites, respectively. (C) Comparison of percentage of methylated CpG sites of $11 \mathrm{CpGs}$ in the OCT4 minimal promoter region and exon 1 region. Student's t-test was used for statistical analysis. ${ }^{*} \mathrm{P}<0.05$.

was correlated with the hypomethylation of promoter and exon. A total of $11 \mathrm{CpG}$ dinucleotides were measured through BSP sequencing. The minimal promoter region and the exon region contained 5 and $6 \mathrm{CpG}$ dinucleotides, respectively (Fig. 3A). DNA methylation of the OCT4 gene was found in all samples. The CpG dinucleotides in the normal group were highly meth- ylated. However, the DNA methylation level was reduced with the upgrading of the glioma malignancy (Fig. 3B). Statistical analysis showed that DNA methylation level in primary gliomas was significantly lower than that in the normal group $(\mathrm{P}<0.05)$. The percent of methylation in high-grade gliomas was significantly lower than that in low-grade ones $(\mathrm{P}<0.05)($ Fig. $3 \mathrm{C})$. 

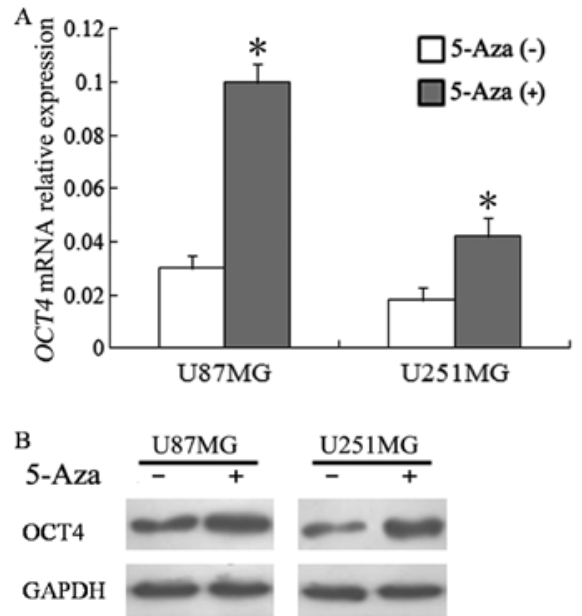

Figure 4. Upregulation of OCT4 expression in U87 and U251 cells following treatment of 5-Aza-dc for $72 \mathrm{~h}$. (A) Real-time qRT-PCR analysis of OCT4 mRNA expression. ${ }^{*} \mathrm{P}<0.05$. (B) Western blot analysis of OCT4 protein expression.

When we compared the OCT4 expression profile (Fig. 1), the DNA methylation level was negatively correlated to the expression of OCT4 mRNA $(r=-0.156, \mathrm{P}<0.05)$.

Upregulation of OCT4 in glioma cells following demethylation. The glioma cell lines (U87MG and U251MG) were treated with 5-Aza-dc, a demethylation reagent, for $72 \mathrm{~h}$. The results showed that OCT4 mRNA expression was upregulated 3.38-fold in U87 cells and 2.35-fold in U251 cells (Fig. 4A). The changing tendency of protein expression was similar to that of mRNA expression (Fig. 4B).

\section{Discussion}

It is considered that most human primary malignant brain tumors contain BTSCs. These cells are the determinants for the occurrence, development and recurrence of gliomas and for the therapeutic response of brain tumor $(29,30)$. BTSCs provide a novel logical explanation for the drug resistance and the high recurrence rate of primary malignant brain tumors. Since BTSCs are similar to neural stem cells in molecular marker expression and multi-lineage differentiation potential, BTSCs can be studied indirectly in the absence of the default marker for screening BTSCs. We hypothesize that the expression level of genes related to stem-like cells (SLCs) can substitute the rate of SLCs in glioma. SLC-related genes usually include Nes (nestin), CD133 (prominin), ABCG2 (ATP-binding cassette superfamily G member 2), SOX2 (SRYbox containing gene 2), OCT4 (POU class 5 homeobox 1) and Msi-1 (musashi-1). In the present study, we selected OCT4 as the representative of SLC-related genes.

During the tumorigenesis of some CSCs in adults, OCT4 expression is detectable since its level is consistent with the number of CSCs and is closely related to the malignant degree, development and prognosis of tumors (31). In this study, real-time PCR, western blot and IHC analyses showed that both OCT4 mRNA and OCT4 protein were highly expressed in primary gliomas and the levels were grade-dependent. Our findings are consistent with previous studies that high- level OCT4 increased the malignancy of ESC-derived tumors $(12-14,31,32)$. Moreover, OCT4 expression is regarded as a 'stem cell survival' factor pattern and is restricted to pluripotent cells $(7,33)$. Our results also supported the BTSC theory of glioma, which suggested that SLCs were present in neoplasm and the malignancy of glioma may be related to the abundance of SLCs in the tumor.

Numerous studies indicate the DNA methylation of the OCT4 gene at the gene regulatory region is a key regulatory factor in OCT4 transcription (22-24,34-36). Cantz et al (37) also argued that the most appropriate method to determine OCT4 expression in cells is to analyze the methylation status of the promoter. Therefore, to explore whether the DNA methylation of OCT4 promoter is involved in the changes of OCT4 expression in primary gliomas, BSP sequencing was employed to measure the methylation of the minimal promoter region and exon region $(-234 \sim+46)$. We found that the methylation levels of the OCT4 gene were different between primary glioma and normal brain tissues. The methylation level in tumor was markedly reduced as compared to the normal group and was lower in high-grade gliomas than in low-grade ones. The results showed that methylation status may regulate the transcription of the OCT4 gene and were involved in glioma development. OCT4 promoter demethylation has already been reported in other types of human cancer. It was also suggested that OCT4 promoter demethylation contributes to tumorigenesis $(38,39)$. Since the demethylation of the OCT4 promter occurred predominantly in high-grade glioma, OCT4 may contribute to the initiation and progression of glioma and potentially serve as a biomarker for the prognosis of human glioma.

Genomic hypomethylation can cause genome instability and proto-oncogene formation, which leads to high expression (40). Our study showed that the expression of the OCT4 gene was significantly upregulated in glioma samples, as compared with the normal brain samples. Notably, the percentage of promoter methylation in the OCT4 gene was inversely correlated with the expression levels of the OCT4 gene in glioma. In vitro, 5-Aza-dc reactivated the expression of OCT4 in glioma cells. These results suggested that aberrant promoter hypomethylation of OCT4 plays a role in the reexpression of its normal silence. Fanelli et al (41) reported that DNA hypomethylation in GBMs is in part explained by a correlating decrease in expression of de novo DNA methyltransferase DNMT3b. This global change in methylation leads to the reactivation and remobilization of repeat elements satellite 2, D4Z4 and Alu elements, resulting in increased copy number alterations of genomic regions in proximity to these elements (42). However, with regard to OCT4, the potential specific mechanism of hypomethylation in primary glioma requires further study.

In summary, OCT4 is epigenetically regulated by methylation in primary glioma. The methylation status of the OCT4 gene can be regulated by a demethylation reagent. Our findings provide evidence for the role of DNA methylation in primary glioma and present a direction for developing more powerful strategies to treat glioma in the clinic.

\section{Acknowledgements}

This study was supported by the Youth Fund of the National Natural Science Foundation of China (81201975), the 
Youth Fund of the Natural Science Foundation of Jiangsu Province (BK2012224), the Natural Science Foundation of China Ministry of Health (2010-2-025), the Natural Science Foundation of Jiangsu Department of Health (H201124), the Six Major Human Resources Project of Jiangsu Province (2011WS-065; 2010-WS-038), the Natural Science Foundation of Jiangsu Colleges and Universities Grant (11KJB320010).

\section{References}

1. Bondy ML, Scheurer ME, Malmer B, et al: Brain tumor epidemiology: consensus from the Brain Tumor Epidemiology Consortium. Cancer 113: 1953-1968, 2008.

2. Yuan X, Curtin J, Xiong Y, et al: Isolation of cancer stem cells from adult glioblastoma multiforme. Oncogene 23: 9392-9400, 2004.

3. Galli R, Binda E, Orfanelli U, et al: Isolation and characterization of tumorigenic, stem-like neural precursors from human glioblastoma. Cancer Res 64: 7011-7021, 2004

4. Singh SK, Hawkins C, Clarke ID, et al: Identification of human brain tumour initiating cells. Nature 432: 396-401, 2004.

5. Hemmati HD, Nakano I, Lazareff JA, et al: Cancerous stem cells can arise from pediatric brain tumors. Proc Natl Acad Sci USA 100: 15178-15183, 2003.

6. Schöler H, Ruppert S, Suzuki N, et al: New type of POU domain in germ line-specific protein Oct-4. Nature 344: 435-439, 1990.

7. Pesce M and Schöler HR: Oct-4: gatekeeper in the beginnings of mammalian development. Stem Cells 19: 271-278, 2001.

8. Takeda J, Seino S and Bell GT: Human Oct3 gene family: cDNA sequences, alternative splicing, gene organization, chromosomal location, and expression at low levels in adult tissues. Nucleic Acids Res 20: 4613-4620, 1992.

9. Babaie Y, Herwig R, Greber B, et al: Analysis of Oct4-dependent transcriptional networks regulating self-renewal and pluripotency in human embryonic stem cells. Stem Cells 25: 500-510, 2007.

10. Webster JD, Yuzbasiyan-Gurkan V, Trosko JE, et al: Expression of the embryonic transcription factor Oct4 in canine neoplasms: a potential marker for stem cell subpopulations in neoplasia. Vet Pathol 44: 893-900, 2007.

11. Chiou SH, Yu CC, Huang CY, et al: Positive correlations of Oct-4 and Nanog in oral cancer stem-like cells and high-grade oral squamous cell carcinoma. Clin Cancer Res 14: 4085-4095, 2008.

12. Du Z, Jia D, Liu S, et al: Oct4 is expressed in human gliomas and promotes colony formation in glioma cells. Glia 57: 724-733, 2009.

13. Holmberg J, He X, Peredo I, et al: Activation of neural and pluripotent stem cell signatures correlates with increased malignancy in human glioma. PloS One 6: e18454, 2011.

14. Ben-Porath I, Thomson MW, Carey VJ, et al: An embryonic stem cell-like gene expression signature in poorly differentiated aggressive human tumors. Nat Genet 40: 499-507, 2008.

15. Ellis L, Atadja PW and Johnstone RW: Epigenetics in cancer: targeting chromatin modifications. Mol Cancer Ther 8: 1409-1420, 2009.

16. Baylin SB and Herman JG: DNA hypermethylation in tumorigenesis: epigenetics joins genetics. Trends Genet 16: 168-174, 2000.

17. Ehrich M, Turner J, Gibbs P, et al: Cytosine methylation profiling of cancer cell lines. Proc Natl Acad Sci USA 105: 4844-4849, 2008.

18. Jones PA: Epigenetics in carcinogenesis and cancer prevention. Ann NY Acad Sci 983: 213-219, 2003.

19. Feinberg AP: Cancer epigenetics takes center stage. Proc Natl Acad Sci USA 98: 392-394, 2001.

20. Nagarajan RP and Costello JF: Molecular epigenetics and genetics in neuro-oncology. Neurotherapeutics 6: 436-446, 2009
21. Shi G and Jin Y: Role of Oct4 in maintaining and regaining stem cell pluripotency. Stem Cell Res Ther 1: 39, 2010.

22. Feldman N, Gerson A, Fang J, et al: G9a-mediated irreversible epigenetic inactivation of Oct-3/4 during early embryogenesis. Nat Cell Biol 8: 188-194, 2006.

23. Deb-Rinker P, Ly D, Jezierski A, et al: Sequential DNA methylation of the Nanog and Oct-4 upstream regions in human NT2 cells during neuronal differentiation. J Biol Chem 280: 6257-6260, 2005.

24. Gidekel S and Bergman Y: A unique developmental pattern of Oct-3/4 DNA methylation is controlled by a cis-demodification element. J Biol Chem 277: 34521-34530, 2002.

25. Hattori N, Nishino K, Ko YG, et al: Epigenetic control of mouse Oct-4 gene expression in embryonic stem cells and trophoblast stem cells. J Biol Chem 279: 17063-17069, 2004.

26. Xue WC, Chan KY, Feng HC, et al: Promoter hypermethylation of multiple genes in hydatidiform mole and choriocarcinoma. J Mol Diagn 6: 326-334, 2004.

27. Liao X, Siu MK, Chan KY, et al: Hypermethylation of RAS effector related genes and DNA methyltransferase 1 expression in endometrial carcinogenesis. Int J Cancer 123: 296-302, 2008.

28. Nordhoff V, Hübner K, Bauer A, et al: Comparative analysis of human, bovine, and murine Oct-4 upstream promoter sequences. Mamm Genome 12: 309-317, 2001.

29. Singh SK, Clarke ID, Terasaki M, et al: Identification of a cancer stem cell in human brain tumors. Cancer Res 63: 5821-5828, 2003.

30. Singh SK, Clarke ID, Hide T and Dirks PB: Cancer stem cells in nervous system tumors. Oncogene 23: 7267-7273, 2004.

31. Sathomsumetee S, Reardon DA, Desjardins A, et al: Molecularly targeted therapy for malignant glioma. Cancer 110: 13-24, 2007.

32. Gidekel S, Pizov G, Bergman Y and Pikarsky E: Oct-3/4 is a dose-dependent oncogenic fate determinant. Cancer Cell 4: 361-370, 2003.

33. Yeom YI, Fuhrmann G, Ovitt CE, et al: Germline regulatory element of Oct- 4 specific for the totipotent cycle of embryonal cells. Development 122: 881-894, 1996.

34. Ben-Shushan E, Pikarsky E, Klar A and Bergman Y: Extinction of Oct-3/4 gene expression in embryonal carcinoma $\mathrm{x}$ fibroblast somatic cell hybrids is accompanied by changes in the methylation status, chromatin structure, and transcriptional activity of the Oct-3/4 upstream region. Mol Cell Biol 13: 891-901, 1993.

35. Simonsson $\mathrm{S}$ and Gurdon J: DNA demethylation is necessary for the epigenetic reprogramming of somatic cell nuclei. Nat Cell Biol 6: 984-990, 2004.

36. Tsuji-Takayama K, Inoue T, Ijiri Y, et al: Demethylating agent, 5-azacytidine, reverses differentiation of embryonic stem cells. Biochem Biophys Res Commun 323: 86-90, 2004.

37. Cantz T, Key G, Bleidissel M, et al: Absence of OCT4 expression in somatic tumor cell lines. Stem Cells 26: 692-697, 2008.

38. Zhang HJ, Siu MK, Wong ES, et al: Oct4 is epigenetically regulated by methylation in normal placenta and gestational trophoblastic disease. Placenta 29: 549-554, 2008.

39. Hoffmann MJ, Müller M, Engers R and Schulz WA: Epigenetic control of CTCFL/BORIS and OCT4 expression in urogenital malignancies. Biochem Pharmacol 72: 1577-1588, 2006.

40. Ehrlich M: DNA methylation in cancer: too much, but also too little. Oncogene 21: 5400-5413, 2002.

41. Fanelli M, Caprodossi S, Ricci-Vitiani L, et al: Loss of pericentromeric DNA methylation pattern in human glioblastoma is associated with altered DNA methyltransferases expression and involves the stem cell compartment. Oncogene 27: 358-365, 2008.

42. Cadieux B, Ching TT, VandenBerg SR and Costello JF: Genome-wide hypomethylation in human glioblastomas associated with specific copy number alteration, methylenetetrahydrofolate reductase allele status, and increased proliferation. Cancer Res 66: 8469-8476, 2006. 